\title{
Patterns of Dynamical Gauge Symmetry Breaking
}

\author{
Ning Chen, Thomas A. Ryttov, and Robert Shrock \\ C. N. Yang Institute for Theoretical Physics \\ Stony Brook University \\ Stony Brook, NY 11794
}

\begin{abstract}
We construct and analyze theories with a gauge symmetry in the ultraviolet of the form $G \otimes G_{b}$, in which the vectorial, asymptotically free $G_{b}$ gauge interaction becomes strongly coupled at a scale where the $G$ interaction is weakly coupled and produces bilinear fermion condensates that dynamically break the $G$ symmetry. Comparisons are given between Higgs and dynamical symmetry breaking mechanisms for various models.

PACS numbers: $12.60 .-\mathrm{i}, 12.60 . \mathrm{Nz}, 11.15 .-\mathrm{q}$
\end{abstract}

\section{INTRODUCTION}

An outstanding question at present concerns the origin of electroweak symmetry breaking (EWSB), in which the electroweak gauge symmetry of the Standard Model $(\mathrm{SM})$, based on the gauge group $G_{E W}=\mathrm{SU}(2)_{L} \otimes \mathrm{U}(1)_{Y}$, where $\mathrm{SU}(2)_{L}$ and $\mathrm{U}(1)_{Y}$ are the factor groups for weak isospin and hypercharge, is broken to the electromagnetic $\mathrm{U}(1)_{e m}$ subgroup. The Standard Model hypothesizes that this symmetry breaking is due to the vacuum expectation value (VEV) of a fundamental Higgs field that transforms as $T=1 / 2$ and $Y=1$. Similarly, the minimal supersymmetric Standard Model (MSSM) attributes electroweak symmetry breaking to nonzero VEVs of the (scalar components of) two Higgs chiral superfields with $T=1 / 2$ and $Y= \pm 1$. A rather different approach is taken by technicolor (TC) theories. In these, the vectorial, asymptotically free technicolor gauge interaction becomes strongly coupled at the $\mathrm{TeV}$ scale, producing condensates of technifermions that break $G_{E W}$ to $\mathrm{U}(1)_{e m}$. Other possibilities have also been studied, such as electroweak symmetry breaking due to boundary conditions on gauge fields in higher dimensions. Experiments at the Large Hadron Collider (LHC) are currently underway to answer the question of the origin of electroweak symmetry breaking.

In general, a comparative study of Higgs-type and dynamical approaches to the breaking of gauge symmetries gives insights into both of these approaches. In this paper we shall carry out such a study. We shall consider a class of gauge theories with a direct-product gauge symmetry of the Lagrangian, of the form

$$
G_{U V}=G \otimes G_{b}
$$

such that as the theory evolves from some high energy scale to lower energies, the $G_{b}$ interaction becomes strongly coupled at a scale $\Lambda_{b}$, where the $G$ interaction is weakly coupled, and produces bilinear fermion condensates that transform as nonsinglets under $G$ and hence dynamically break the $G$ symmetry to a subgroup $H \subset G$, i.e.,

$$
G \rightarrow H \quad \text { induced by } G_{b} \text {. }
$$

(The subscript $b$ on $G_{b}$ and $\Lambda_{b}$ refers to their roles in the breaking of $G$.) The condition that the $G$ interaction is weakly coupled at the scale $\Lambda_{b}$ is similar to the fact that the electroweak interaction is weakly coupled at the scale $2^{-1 / 4} G_{F}^{-1 / 2} \simeq 250 \mathrm{GeV}$ where it is broken. However, our study is not an attempt to construct a semi-realistic theory of dynamical EWSB, but instead focuses on gaining insights into the differences between Higgs-type and dynamical symmetry breaking through comparative analyses of various models.

In order for the dynamical symmetry in Eq. (1.2) to occur, the following conditions are necessary and are therefore assumed here: (i) the $G_{b}$ gauge interaction is asymptotically free, so that the running coupling $\alpha_{b}(\mu)=g_{b}(\mu)^{2} /(4 \pi)$ increases as the reference energy scale $\mu$ decreases; (ii) $G_{b}$, considered by itself, is a vectorial gauge symmetry, so that it does not self-break when it forms condensates, but instead remains exact; and (iii) the content of fermions that are nonsinglets under $G_{b}$ is sufficiently small so that as the $G_{b}$ interaction evolves from the ultraviolet to lower energy scales, $\alpha_{b}(\mu)$ increases sufficiently to exceed the critical value for the formation of the requisite $G$-breaking fermion condensates rather than evolving in a chirally symmetric manner. We consider several types of symmetries $G$, both vectorial and chiral, and of both direct-product and (semi)simple type. Although $G_{b}$, considered by itself (with the $G$ interaction turned off), is vectorial, the full gauge symmetry $G_{U V}$ is chiral in all of the cases that we consider. The $G_{U V}$ symmetry thus requires that the fermions that are nonsinglets under $G_{b}$ have zero intrinsic masses.

One can generalize the analysis further to deal with gauge symmetries of the form

$$
G_{U V}=G \otimes\left[\prod_{i=1}^{k} G_{b_{i}}\right],
$$

where $k$ strongly coupled gauge interactions $G_{b_{i}}, 1 \leq i \leq$ $k$, play a role in the dynamical breaking of $G$. We will focus on the simplest case, $k=1$, but will also comment on models with $k=2$.

This paper is organized as follows. In Sect. II we review two illustrative examples of the type of induced gauge symmetry breaking that we consider. In Sect. III 
we carry out a comparative study of the breaking of an $\mathrm{SU}(3)$ gauge symmetry to $\mathrm{SU}(2)$ by a Higgs field in the fundamental representation and by a dynamical mechanism. We also discuss how color $\mathrm{SU}(3)_{c}$ would be broken in a modified Standard Model with a strongly coupled $\mathrm{SU}(2)_{L}$ interaction. In Sect. IV we carry out a comparative study of the breaking of an $\mathrm{SU}(3)$ gauge symmetry by a Higgs field in the adjoint representation and by a dynamical mechanism. This is generalized to $\mathrm{SU}(N)$ in Sect. V. Some further discussion and our conclusions are given in Sects. VI and VII.

\section{SOME EXAMPLES OF INDUCED DYNAMICAL SYMMETRY BREAKING}

\section{A. QCD Breaking Electroweak Symmetry}

As background for our work, we first briefly review two examples of induced dynamical symmetry breaking of weakly coupled gauge symmetries by strongly coupled gauge interactions. In addition to the physics that is responsible for the main electroweak symmetry breaking at the scale $\sim 250 \mathrm{GeV}$, there is another source of EWSB, albeit at a much smaller mass scale. This is quantum chromodynamics (QCD). The color $\mathrm{SU}(3)_{c}$ gauge interaction produces bilinear quark condensates at a scale $\Lambda_{Q C D} \sim 250 \mathrm{MeV}$, in the most attractive channel (MAC) $3 \times \overline{3} \rightarrow 1$, of the form $\langle\bar{q} q\rangle=\left\langle\bar{q}_{L} q_{R}\right\rangle+$ h.c. Because these quark condensates transform as weak $T=1 / 2$, $|Y|=1$ quantities, they break $G_{E W}$ to electromagnetic $\mathrm{U}(1)_{\mathrm{em}}$. Indeed, one could imagine a hypothetical world in which the electroweak symmetry were not broken at the normal scale, but instead remained valid all the way down to the QCD scale. In this world (assuming that the $\mathrm{SU}(3)_{c}, \mathrm{SU}(2)_{L}$, and $\mathrm{U}(1)_{Y}$ running gauge couplings had approximately their usual values), QCD would be the main source of EWSB [1, 2]. Such a theory would be of the form of Eqs. (1.1) and (1.2), with

$$
G=G_{E W}, \quad G_{b}=\mathrm{SU}(3)_{c}, \quad H=\mathrm{U}(1)_{e m} .
$$

In this hypothetical world the $W$ and $Z$ would pick up masses given by $m_{W}^{2}=g^{2} f_{\pi}^{2} / 4$ and $m_{Z}^{2}=\left(g^{2}+g^{\prime 2}\right) f_{\pi}^{2} / 4$, where $g$ and $g^{\prime}$ are the $\mathrm{SU}(2)_{L}$ and $\mathrm{U}(1)_{Y}$ running gauge couplings at the scale $\Lambda_{Q C D}$, and $f_{\pi}$ is the pion decay constant.

\section{B. Electroweak Symmetry Breaking by Technicolor}

Technicolor models embody the idea of dynamical electroweak symmetry breaking [1] (recent reviews include [3]). In these models, the gauge symmetry that is broken is (the electroweak part of) the SM gauge group $G=G_{S M}=\mathrm{SU}(3)_{c} \otimes S U(2)_{L} \otimes \mathrm{U}(1)_{Y}$. At the scale where $G_{S M}$ is broken, all of the three gauge interactions corresponding to its factor groups are weakly coupled.
The technicolor gauge interaction is associated with the group $G_{b}=G_{T C}$. Typically, $G_{T C}=\mathrm{SU}\left(N_{T C}\right)$ with some value of $N_{T C}$ such as 2 , so these models can be described in the notation of Eqs. (1.1) and (1.2) by

$G=G_{S M}, \quad G_{b}=\mathrm{SU}\left(N_{T C}\right), \quad H=\mathrm{SU}(3)_{c} \otimes \mathrm{U}(1)_{e m}$.

The (vectorial, asymptotically free) technicolor gauge interaction produces condensates of technifermions $\langle\bar{F} F\rangle=$ $\left\langle\bar{F}_{L} F_{R}\right\rangle+$ h.c. that transform as weak $T=1 / 2,|Y|=1$ and hence break $G_{E W}$ to $\mathrm{U}(1)_{e m}$, as indicated in Eq. (2.2). Technicolor models are embedded in extended technicolor (ETC) in order to communicate the electroweak symmetry breaking to the quarks and leptons. These TC/ETC theories are subject to a number of constraints from induced flavor-changing neutral processes, precision electroweak data, and limits on pseudo-NambuGoldstone bosons (PNGBs).

Technicolor models can be classified into two generic types: (i) one-family models, in which the technifermions comprise one SM family, and (ii) one-doublet models, in which, among the technifermions, there is only a single electroweak doublet. One-family (but not one-doublet) technicolor models feature a color-octet technivector meson resonance, as well as a color-nonsinglet PNGB's. Many searches for technihadrons have been carried out 3]. Recent LHC results from the ATLAS and CMS experiments have set lower limits of order $1.5 \mathrm{TeV}$ on a color-octet technivector meson [4, 5].

\section{BREAKING AN SU(3) GAUGE SYMMETRY TO SU(2)}

In this section we shall compare Higgs and dynamical mechanisms for breaking an $\mathrm{SU}(3)$ gauge symmetry to $\mathrm{SU}(2)$. We assume that the fermion content of the theory is such that the fermions that are only nonsinglets under $\mathrm{SU}(3)$ form a vectorlike sector. We shall begin by considering an abstract asymptotically free $\mathrm{SU}(3)$ theory at a sufficiently high scale that it is weakly coupled.

\section{A. Higgs Mechanism to break SU(3) to SU(2)}

The requisite breaking can be accomplished by including a Higgs field $\phi$ that transforms as a fundamental (triplet) representation of the $\mathrm{SU}(3)$ group, with a potential

$$
V=\frac{\mu^{2}}{2} \phi^{\dagger} \phi+\frac{\lambda}{4}\left(\phi^{\dagger} \phi\right)^{2},
$$

where $\mu^{2}<0$ (and $\lambda>0$ in order for $V$ to be bounded from below). This potential is minimized for a nonzero value of the $\phi$ vacuum expectation value. Without loss of generality, one can use the $\mathrm{SU}(3)$ gauge invariance to define directions in $\mathrm{SU}(3)$ space so that this has the form 
$\langle\phi\rangle=(0,0,1)^{T} v$, and one can perform a global phase redefinition on $\phi$ to make $v$ real. This breaks $\mathrm{SU}(3)$ to the $\mathrm{SU}(2)$ subgroup generated by the the $\mathrm{SU}(3)$ generators $T_{a}$ with $a=1,2,3$ (in the usual Gell-Mann ordering of these generators). Of the six real components of the $\phi$ field, five are Nambu-Goldstone bosons and are absorbed by the five gauge bosons in the coset space $\mathrm{SU}(3) / \mathrm{SU}(2)$ to form the longitudinal polarization states of the resultant massive vector bosons. The resultant vector boson masses are $\propto g_{3}(v) v$, where $g_{3} \equiv g_{3}(\mu)$ is the running $\mathrm{SU}(3)$ gauge coupling at the scale $\mu=v$. The sixth component of the $\phi$ field forms a physical Higgs boson with a mass $\sim \sqrt{\lambda} v$. This is a singlet under the residual $\mathrm{SU}(2)$ gauge interaction.

As noted above, we assume that this breaking occurs at a scale $v$ that is large compared with the scale where the running $\mathrm{SU}(3)$ gauge coupling $\alpha_{3}(\mu)=g_{3}(\mu)^{2} /(4 \pi)$ would have grown to $\mathrm{O}(1)$ and the theory would thus have become strongly coupled. This assumption is necessary for this model to fall under the class of theories that are considered in this paper. If one were to relax this assumption, the analysis would become more complicated, because one would not be able to perform a perturbative analysis of the Higgs sector. (For a recent discussion of this strongly coupled case and further references, see [6]). Below the scale $v$, the resultant $\mathrm{SU}(2)$ theory would have a fermion sector consisting of the $\mathrm{SU}(2)$-nonsinglet components of the original $\mathrm{SU}(3)$ fermion sector, together with the $\mathrm{SU}(2)$ gluons, with a gauge coupling inherited from the original SU(3) theory. This SU(2) theory would then evolve further into the infrared. With a sufficiently small fermion content $\{f\}$, the $\mathrm{SU}(2)$ coupling would eventually increase to $O(1)$ at a lower scale $\Lambda_{2}$, where the $\mathrm{SU}(2)$ interaction would confine and produce bilinear fermion condensates. There would thus be a spectrum of SU(2)-singlet meson and (bosonic) baryons, together with glueballs (which would mix with the mesons to produce mass eigenstates) at this lower scale $\Lambda_{2}$.

There are several properties of this Higgs mechanism that will be contrasted with the induced dynamical breaking mechanism to be discussed next. First, a priori, one has the freedom to choose the coefficient $\mu^{2}$ in the Higgs potential (3.1) to be positive or negative. Since one wants to construct the Higgs mechanism to break SU(3), one chooses $\mu^{2}<0$, but this sign choice could be considered to be ad hoc, since one does not give any deeper explanation for this choice. Second, the Higgs mechanism predicts physical pointlike Higgs particle(s), whereas in a dynamical mechanism, although the $G_{b}$ interaction leads to various $G_{b}$-singlet bound states, including some with angular momentum $J=0$, the properties of these states are not, in general, the same as those of a Higgs particle. Third, as is well known, this potential is unstable to large loop corrections and is thus sensitive to the nature of the ultraviolet completion of the theory (i.e., has a hierarchy problem). A fourth and related point is that the Higgs sector is not asymptotically free, i.e., the beta function for the quartic coupling, $d \lambda / d t$, is positive, where $t=\ln \mu$. Because of this, if one fixes $\lambda$ at the scale $v$, say, then one must worry about a possible Landau pole in $\lambda$ that could occur at a scale $\mu>>v$. An equivalent way to phrase this is that if one fixes $\lambda$ at a high scale in the ultraviolet, then $\lambda$ decreases as $\mu$ decreases and is subject to an upper bound at a much lower scale such as $v$ [7].

\section{B. Induced Dynamical Breaking of SU(3) to SU(2)}

In this subsection we discuss how one can produce the breaking of the $\mathrm{SU}(3)$ symmetry to $\mathrm{SU}(2)$ in a dynamical manner. For $G_{b}$ we choose the smallest non-Abelian Lie group, $\mathrm{SU}(2)_{b}$, so that $G_{U V}=\mathrm{SU}(3) \otimes \mathrm{SU}(2)_{b}$, in the notation of Eq. (1.1). To the set of fermions $\{f\}$ transforming vectorially under $\mathrm{SU}(3)$ we add the following chiral fermions (where $a$ and $\alpha$ denote SU(3) and $\mathrm{SU}(2)_{b}$ gauge indices, respectively and the numbers in parenthese denote the dimensionalities of the representations of $\left.G_{U V}\right)$ : (i) $\zeta_{L}^{a \alpha} \quad:(3,2)$; (ii) $\eta_{L}^{\alpha}:(1,2)$; and (iii) $\chi_{p, R}^{a}:(3,1)$ with $p=1,2$. This set of fermions is similar to the set that one of us used in Ref. [6]. Since the $\mathrm{SU}(2)_{b}$ gauge interaction is asymptotically free, as the reference energy scale $\mu$ decreases from large values, the running coupling $\alpha_{b}(\mu)$ increases. The $\mathrm{SU}(2)_{b^{-}}$ nonsinglet fermions comprise four chiral Weyl fermions or equivalently, two Dirac fermions. This is well below the estimated critical number $N_{f, c r} \sim 8$ beyond which the $\mathrm{SU}(2)_{b}$ theory would evolve into the infrared in a chirally symmetric manner [8]. Therefore, we can conclude that as $\mu$ decreases to the scale $\mu=\Lambda_{b}$ such that $\alpha_{b}(\mu) \sim O(1)$, the $\mathrm{SU}(2)_{b}$ interaction produces bilinear fermion condensates. The most attractive channel for the fermion condensation is $2 \times 2 \rightarrow 1$. One such condensate is of the form $\left\langle\epsilon_{\alpha \beta} \zeta_{L}^{a \alpha}{ }^{T} C \zeta_{L}^{b \beta}\right\rangle$, where $\epsilon_{\alpha \beta}$ is the antisymmetric tensor density for $\mathrm{SU}(2)_{b}$. This is automatically antisymmetrized in the $\mathrm{SU}(3)$ indices $a, b$ and hence is proportional to

$$
\left\langle\epsilon_{a b c} \epsilon_{\alpha \beta} \zeta_{L}^{a \alpha T} C \zeta_{L}^{b \beta}\right\rangle
$$

where $\epsilon_{a b c}$ is the antisymmetric tensor density for $\mathrm{SU}(3)$. The condensate (3.2) transforms as conjugate fundamental ( $\overline{3})$ representation of SU(3) and therefore dynamically breaks $\mathrm{SU}(3)$ to an $\mathrm{SU}(2)$ subgroup. A second condensate formed by the $\mathrm{SU}(2)_{b}$ interaction is

$$
\left\langle\epsilon_{\alpha \beta} \zeta_{L}^{a \alpha T} C \eta_{L}^{\beta}\right\rangle
$$

This transforms as a fundamental representation of $\mathrm{SU}(3)$ and hence also breaks it to an $\mathrm{SU}(2)$ subgroup. One can use vacuum alignment arguments [9] to infer that these $\mathrm{SU}(2)$ subgroups are the same. Then, without loss of generality, one may choose the index $c=3$ in the condensate (3.2) and $a=3$ in the condensate (3.3). The residual SU(2) subgroup preserved by these condensates is thus the one generated by $T_{a}, a=1,2,3$ in $\mathrm{SU}(3)$. The fermions $\zeta_{L}^{a}{ }^{\alpha}$ and $\eta_{L}^{\alpha}$ with $a=1,2,3, \alpha=1,2$ involved 
in these condensates gain dynamical masses of order $\Lambda_{b}$ and are integrated out of the low-energy effective field theory that is operative as scales $\mu<\Lambda_{2}$. The two copies of $\chi_{p, L}^{a}$ decompose as two doublets under the resultant $\mathrm{SU}(2)$ for $a=1,2$ (while the $a=3$ components form two singlets). The fermion content of this low-energy $\mathrm{SU}(2)$ theory thus consists of these two doublets, together with the $\mathrm{SU}(2)$-nonsinglet components of the set $\{f\}$. With an asymptotically free $\mathrm{SU}(2)$, the coupling $\alpha_{2}(\mu)$, which is inherited from the weakly coupled SU(3) theory will increase as $\mu$ decreases below $\Lambda_{b}$, and if the fermion content is sufficiently small so that $\alpha_{2}(\mu)$ grows to $\mathrm{O}(1)$ at a lower scale $\Lambda_{2}$, the $\mathrm{SU}(2)$ gauge interaction will confine and form bilinear fermion condensates at this scale. Given that $\alpha_{2}$ is small at the scale $\Lambda_{b}$ and increases only logarithmically, it follows that $\Lambda_{2}<<\Lambda_{b}$.

If one were to turn off the $\mathrm{SU}(3)$ gauge interaction, the $\mathrm{SU}(2)_{b}$ theory would have a classical $\mathrm{U}(4)$ or equivalently $\mathrm{SU}(4) \otimes \mathrm{U}(1)$ global chiral symmetry. The $\mathrm{U}(1)$ is broken by $\mathrm{SU}(2)_{b}$ instantons, so that the actual nonanomalous global chiral symmetry would be the SU(4) (generated by global transformations of the $\zeta_{L}^{a}{ }^{\alpha}$ and $\eta_{L}^{\alpha}$ among each other for a fixed $\alpha$.) The bilinear condensates would break this to $\mathrm{Sp}(4)$, with the resultant appearance of five Nambu-Goldstone bosons. Turning on the SU(3) gauge interaction explicitly breaks this global symmetry, although the breaking is weak, since $\alpha_{3}$ is small.

We contrast this dynamical breaking with the corresponding Higgs mechanism presented above. First, the dynamical mechanism is more predictive, in the sense that once one has specified the gauge interaction $G_{b}$ and the fermion content, the resulting fermion condensation and symmetry breaking follow automatically; one does not have to make an ad hoc choice of a parameter, as one does with the choice $\mu^{2}<0$ in the Higgs potential (3.1). Second, the theory does not suffer from a hierarchy problem, i.e., is not sensitively dependent on an ultraviolet completion, in contrast to the Higgs mechanism. Third, by construction, both the $\mathrm{SU}(3)$ and the $\mathrm{SU}(2)_{b}$ sectors are asymptotically free, again in contrast with the Higgs mechanism, in which the quartic coupling is not asymptotically free.

\section{Induced Breaking of $\mathrm{SU}(3)_{c}$ in a Modified Standard Model}

Here we discuss another way to break an $\mathrm{SU}(3)$ gauge symmetry dynamically. In this case we will take the $\mathrm{SU}(3)$ to be the color $\mathrm{SU}(3)_{c}$ group of the Standard Model. The point here is that with a modification of the properties of the Standard Model, color SU $(3)_{c}$ would, in fact, be dynamically broken by the $\mathrm{SU}(2)_{L}$ gauge interaction. Our analysis also addresses a fundamental question in particle physics. One of the profound properties of nature is the fact that it is the chiral part of $G_{S M}$ that is broken, leaving as a residual exact subgroup a symmetry that is vectorial, namely $H=\mathrm{SU}(3)_{c} \otimes \mathrm{U}(1)_{e m}$. This is naturally explained in the Standard Model Higgs mechanism and also in technicolor theories. One is led, then, to ask how general this property is in quantum field theory. That is, can one construct a model that exhibits dynamical breaking of a vectorial gauge symmetry? Clearly, this requires more than one gauge interaction to be present, since if one has just a single vectorial gauge interaction and it becomes strongly coupled and produces condensates, then the most attractive channel is $R_{i} \times \bar{R}_{i} \rightarrow 1$ for the one or more fermion representations $R_{i}$ in the theory, so it does not self-break [10, 11].

Let us thus consider a theory with the same gauge group, $G_{S M}$, but make two changes: (i) first, we remove the usual breaking of $\mathrm{SU}(2)_{L}$ at the $250 \mathrm{GeV}$ scale, and (ii) we arrange the values of the gauge couplings so that at a scale $\Lambda_{2}$ considerably larger than $\Lambda_{Q C D}$, where $\mathrm{SU}(3)_{c}$ (and $\left.\mathrm{U}(1)_{Y}\right)$ are weakly coupled, the $\mathrm{SU}(2)_{L}$ interaction becomes strongly coupled, with $\alpha_{2}\left(\Lambda_{2}\right)=g\left(\Lambda_{2}\right)^{2} /(4 \pi)$ of order unity. The $\mathrm{SU}(2)_{L}$ sector contains $N_{\text {gen }}\left(N_{c}+1\right)=12$ chiral fermion doublets (where $N_{\text {gen }}$ denotes the number of SM fermion generations), so that the $\mathrm{SU}(2)_{L}$ gauge interaction is asymptotically free, with leading coefficient

$$
\left(b_{1}\right)_{S U(2)_{L}}=\frac{1}{3}\left[22-\left(N_{c}+1\right) N_{g e n}\right] .
$$

Given that there is no breaking of $G_{E W}$, the fermions are massless, so they all contribute to the $\mathrm{SU}(2)_{L}$ beta function. To illustrate this dynamical breaking in the simplest context, we assume $N_{\text {gen }}=1$, so that there are four chiral SU $(2)_{L}$ doublets, or equivalently, $N_{f}=2$ Dirac doublets. This is well within the phase in which $\mathrm{SU}(2)_{L}$ confines and spontaneously breaks global chiral symmetry. The model thus contains one family of SM fermions: $Q_{L}^{a i}=\left(\begin{array}{c}u^{a} \\ d^{a}\end{array}\right)_{L}, u_{R}^{a}, d_{R}^{a}, L_{L}^{i}=\left(\begin{array}{c}\nu_{e} \\ e\end{array}\right)_{L}$, and $e_{R}$, where $a$ and $i$ are $\mathrm{SU}(3)_{c}$ and $\mathrm{SU}(2)_{L}$ gauge indices, respectively.

The most attractive channel for the strongly coupled $\mathrm{SU}(2)_{L}$ interaction is $2 \times 2 \rightarrow 1$, and it produces several condensates in this channel. The first of these is of the form $\left\langle\epsilon_{k \ell} Q_{L}^{a, k T} C Q_{L}^{b, \ell}\right\rangle$, where $\epsilon_{k \ell}$ is the antisymmetric tensor density for $\mathrm{SU}(2)_{L}$. This is automatically antisymmetric in $\mathrm{SU}(3)_{c}$ indices and hence is proportional to

$$
\left\langle\epsilon_{a b c} \epsilon_{k \ell} Q_{L}^{a, k T} C Q_{L}^{b, \ell}\right\rangle=2\left\langle\epsilon_{a b c} u_{L}^{a}{ }^{T} C d_{L}^{b}\right\rangle,
$$

This transforms as a $(3 \times 3)_{a s}=\overline{3}$ under $\mathrm{SU}(3)_{c}$ (where the subscript as stands for antisymmetric) and hence breaks $\mathrm{SU}(3)_{c}$ to a subgroup $\mathrm{SU}(2)_{c}$. It also breaks $\mathrm{U}(1)_{Y}$. As is clear from the fact that electric charge satisfies $Q=T_{3}+(Y / 2)$ and the fact that the condensate (3.5) is invariant under $\mathrm{SU}(2)_{L}$, it also violates electric charge invariance. Without loss of generality, we choose the breaking direction of $\mathrm{SU}(3)_{c}$ as the third direction, so that the $u_{L}^{a}$ and $d_{L}^{a}$ quarks with color indices $a=1,2$ occur in the condensate (3.5) and hence gain dynamical masses of order $\Lambda_{2}$. 
The strong $\mathrm{SU}(2)_{L}$ interaction would also produce the condensate

$$
\left\langle\epsilon_{k \ell} Q_{L}^{a, k T} C L_{L}^{\ell}\right\rangle=\left\langle u_{L}^{a T} C e_{L}-d_{L}^{a}{ }^{T} C \nu_{e, L}\right\rangle .
$$

This also breaks $\mathrm{SU}(3)_{c}$ to an $\mathrm{SU}(2)_{c}$ subgroup and violates hypercharge and electric charge. As in our discussion above, a vacuum alignment argument can be used to infer that the condensate (3.6) breaks $\mathrm{SU}(3)_{c}$ to the same $\mathrm{SU}(2)_{c}$ as the condensate (3.5), so that the color index in Eq. (3.6) has the value $a=3$. This $\mathrm{SU}(2)_{c}$ is the one generated by the color generators $\left(T_{a}\right)$ with $a=1,2,3$. Thus, this model is of the form in Eqs. (1.1) and (1.2) with

$$
G=\mathrm{SU}(3)_{c} \otimes \mathrm{U}(1)_{Y}, \quad G_{b}=\mathrm{SU}(2)_{L}, \quad H=\mathrm{SU}(2)_{c} .
$$

In addition to breaking these gauge symmetries, the condensate (3.5) breaks baryon number by $\Delta B=2 / 3$, while the condensate (3.6) breaks $B$ by $\Delta B=1 / 3$ and lepton number $L$ by $\Delta L=1$. The quarks $u_{L}^{a}, d_{L}^{a}$ with $a=1,2,3$ and the leptons $e_{L}$, and $\nu_{e, L}$ involved in these condensates gain dynamical masses of order $\Lambda_{2}$. (The actual mass eigenstates involve linear combinations of these fields.) Similarly, the five gluons in the coset $\mathrm{SU}(3)_{c} / \mathrm{SU}(2)_{c}$ corresponding to the broken generators of $\mathrm{SU}(3)_{c}$ gain dynamical masses of order

$$
m_{g} \sim g_{3}\left(\Lambda_{2}\right) \Lambda_{2}
$$

and the abelian $\mathrm{U}(1)_{Y}$ gauge boson $B$ gains a mass

$$
m_{B} \sim g^{\prime}\left(\Lambda_{2}\right) \Lambda_{2} .
$$

Since by our assumptions, $\mathrm{SU}(3)_{c}$ and $\mathrm{U}(1)_{Y}$ are weakly coupled at this scale, the masses of these five gluons and of the one $B$ boson are smaller than the dynamically produced fermion masses.

Of the quarks and leptons in this $N_{\text {gen }}=1$ model, all of the components of the $N_{c}+1=4 \mathrm{SU}(2)_{L}$ doublets are involved in the condensates (3.5) and (3.6) and gain dynamical masses of order $\Lambda_{2}$. These fermions are thus integrated out of the low-energy effective theory below $\Lambda_{2}$. The $\mathrm{SU}(2)_{c}$ gauge symmetry of this low-energy effective field theory remains exact. The content of nonsinglet fermions in this low-energy theory consists of $u_{R}^{a}$ and $d_{R}^{a}$ with $a=1,2$, which form two Weyl fermions, or equivalently, one Dirac fermion. The $\mathrm{SU}(2)_{c}$ gauge coupling $\alpha_{2 c}$ is inherited from the $\mathrm{SU}(3)_{c}$ theory and is small at $\Lambda_{2}$, but eventually grows to $\mathrm{O}(1)$ at a much lower $\Lambda_{2 c}<<\Lambda_{2}$. At this lower scale $\Lambda_{2 c}$, the $\mathrm{SU}(2)_{c}$ theory confines and produces a bilinear fermion condensate,

$$
\left\langle\epsilon_{a b} u_{R}^{a T} C d_{R}^{b}\right\rangle,
$$

where here $\epsilon_{a b}$ is the antisymmetric tensor density of $\mathrm{SU}(2)_{c}$. This $\mathrm{SU}(2)_{c}$ theory has a classical $\mathrm{U}(2)$, or equivalently, $\mathrm{SU}(2) \otimes \mathrm{U}(1)$ global chiral symmetry defined by transformations that mix up the $u_{R}^{a}$ and $d_{R}^{a}$ fields (for fixed $a)$. The $\mathrm{U}(1)$ is broken by $\mathrm{SU}(2)_{c}$ instantons, so that the actual non-anomalous global chiral symmetry is $\mathrm{SU}(2)$. In general, an $\mathrm{SU}(2)$ gauge theory with $N_{d}$ massless chiral Weyl fermions transforming according to the fundamental representation (with $N_{d}=2 k$ even to avoid a global Witten anomaly) has an $\mathrm{SU}(2 k)$ global chiral symmetry corresponding to transformations that mix up the $2 k$ chiral doublets. Formation of condensates involving these doublets breaks this global symmetry to $\mathrm{Sp}(2 k)$. Since the orders of these groups are $4 k^{2}-1$ and $k(2 k+1)$, respectively, this entails the breaking of $2 k^{2}-k-1$ generators of $\mathrm{SU}(2 k)$, and the resultant appearance of this number of massless Nambu-Goldstone bosons. In this $\mathrm{SU}(2)_{c}$ theory, there are $N_{d}=2$ chiral fermions, i.e., $k=1$, so the $\mathrm{SU}(2)$ global chiral symmetry is equivalent to $\operatorname{Sp}(2)$, and there is no chiral symmetry breaking due to the formation of the condensate (3.10).

It is also worthwhile to comment on the situation concerning global chiral symmetry at the higher scale, above $\Lambda_{2}$. In the present model with its one generation of SM fermions, if one turns off the $\mathrm{SU}(3)_{c}$ and $\mathrm{U}(1)_{Y}$ couplings, then, an an energy above $\Lambda_{2}$, the $\mathrm{SU}(2)_{L}$ theory has a non-anomalous global $\mathrm{SU}\left(N_{d}\right)$ symmetry, where $N_{d}=N_{c}+1=4$. The condensates (3.5) and (3.6) break this to $\mathrm{Sp}(4)$, leading to the appearance of five Nambu-Goldstone bosons. Since the NGBs couple in a derivative manner, their scattering amplitudes are suppressed by powers of center-of-mass energy $\sqrt{s} / \Lambda_{2}$ and hence they are progressively more weakly coupled as the energy scale decreases further below $\Lambda_{2}$ [13]. Turning on the $\mathrm{SU}(3)_{c}$ and $\mathrm{U}(1)_{Y}$ couplings explicitly breaks the global SU(4) symmetry, but also the would-be NGBs are absorbed to form the longitudinal components of the five vector bosons in the coset $\mathrm{SU}(3)_{c} / \mathrm{SU}(2)_{c}$. This process is reminiscent of the mechanism by which technicolor gives masses to the $W$ and $Z$ bosons.

Our analysis here answers the question that we posed at the beginning of this subsection concerning the breaking of a vectorial, as contrast to a chiral, gauge symmetry. Our answer is that it is certainly possible for a vectorial gauge symmetry to be broken, if this breaking is induced by another strongly coupled interaction. The reason that $\mathrm{SU}(2)_{L}$ does not break $\mathrm{SU}(3)_{c}$ in nature is a consequence of the fact that $\mathrm{SU}(2)_{L}$ is broken well above the scale where its coupling would have become large enough to produce the condensates (3.5) and (3.6). The resultant $W$ and $Z$ are massive and weakly coupled and their interactions are too weak to induce such condensates. Indeed, even if the $\mathrm{SU}(2)_{L}$ symmetry were not broken at this higher scale, it would be broken by the quark condensates at the QCD scale, as discussed above, before it could become strong enough to break $\mathrm{SU}(3)_{c}$. 


\section{INDUCED DYNAMICAL BREAKING OF A GAUGE SYMMETRY BY ADJOINT FIELDS: AN ILLUSTRATIVE MODEL WITH $G=\mathrm{SU}(3)$}

\section{A. Higgs Mechanism}

We next consider induced breaking of a gauge symmetry by fields that transform as the adjoint representation of the gauge group. In this section we discuss $\mathrm{SU}(3)$ because of some special properties that it has, and in the next section we discuss $\mathrm{SU}(N)$ for general $N \geq 4$. We begin by constructing a Higgs mechanism for this breaking. We assume that the theory contains a Higgs field $\phi$ transforming according to the adjoint (i.e., octet) representation of $\mathrm{SU}(3)$, with an appropriate Higgs potential. We will write the components of $\phi$ as $\phi_{j}^{i}, 1 \leq i, j \leq 3$; these are subject to the trace condition $\operatorname{Tr}(\phi)=\sum_{i=1}^{3} \phi_{i}^{i}=0$. In general, when using the adjoint representation of $\mathrm{SU}(N)$, in addition to the notation $\phi_{j}^{i}$ with $1 \leq i, j \leq N$, it will also be convenient to use an equivalent notation $\phi_{a}$, with $1 \leq a \leq N^{2}-1$, that indicates the 1-1 correspondence with the $N^{2}-1$ generators $T_{a}$ of $\mathrm{SU}(N)$. Thus the $\phi_{j}^{i}$ form the entries of a matrix given by $\sqrt{2} \sum_{a=1}^{N^{2}-1} \phi_{a} T_{a}$.

We will require that the Higgs part of the Lagrangian be invariant under the replacement $\phi \rightarrow-\phi$. It follows that the Higgs potential contains only quadratic and quartic terms in $\phi$. For a general $\mathrm{SU}(N)$ theory with a Higgs field in the adjoint representation, there are two independent quartic terms, proportional to $\left[\operatorname{Tr}\left(\phi^{2}\right)\right]^{2}$ and $\operatorname{Tr}\left(\phi^{4}\right)$. For the special values $N=2$ or $N=3$, $\left[\operatorname{Tr}\left(\phi^{2}\right)\right]^{2}=2 \operatorname{Tr}\left(\phi^{4}\right)$, so there is only one independent quartic term. For the present case of $\mathrm{SU}(3)$, the Higgs potential may thus be written as

$$
V=\frac{\mu^{2}}{2} \operatorname{Tr}\left(\phi^{2}\right)+\frac{\lambda}{4}\left[\operatorname{Tr}\left(\phi^{2}\right)\right]^{2} .
$$

Here we take $\mu^{2}<0$ to get the symmetry breaking. This potential is minimized for a Higgs field vacuum expectation value $(\mathrm{VEV})$ of the form

$$
\langle\phi\rangle=T_{8} v
$$

where $v$ can be made real by a global rephasing of $\phi$ and $T_{8}$ is the second member of the Cartan subalgebra of $\mathrm{SU}(3)$,

$$
T_{8}=\frac{1}{2 \sqrt{3}}\left(\begin{array}{ccc}
1 & 0 & 0 \\
0 & 1 & 0 \\
0 & 0 & -2
\end{array}\right)
$$

The VEV (4.2) breaks SU(3) according to the pattern

$$
\mathrm{SU}(3) \rightarrow \mathrm{SU}(2) \otimes \mathrm{U}(1) .
$$

Since $\mathrm{SU}(3)$ has order eight, while $\mathrm{SU}(2) \otimes \mathrm{U}(1)$ has order four, there are four broken generators of SU(3), namely the $T_{a}$ with $a=4,5,6,7$ in the standard Gell-Mann basis. The corresponding components $\phi_{a}$ are NambuGoldstone bosons and are absorbed to become the longitudinal components of the massive vector bosons. Four physical Higgs fields remain, with masses $\sim \sqrt{\lambda} v$. Of these, $\phi_{a}, a=1,2,3$ transform as the adjoint representation of the residual $\mathrm{SU}(2)$ gauge interaction and, assuming that it confines, they are thus confined in $\mathrm{SU}(2)-$ singlet bound states. Since we have assumed that the $\mathrm{SU}(2)$ theory is weakly coupled at the scale $\mu=v$, and since its coupling increases only logarithmically, the $\mathrm{SU}(2)$ confinement scale $\Lambda_{2}$ is much smaller than $v$. In passing, we note that although a Higgs VEV of the form $\phi=\operatorname{diag}(a, b,-(a+b))$, with $|a| \neq|b|$ is, a priori, possible, and would break $\mathrm{SU}(3)$ to $\mathrm{U}(1)$ rather than $\mathrm{SU}(2) \otimes \mathrm{U}(1)$, it does not minimize the Higgs potential.

\section{B. Dynamical Breaking Mechanism with Adjoint Fields}

To study the dynamical breaking of the SU(3) symmetry by an $\mathrm{SU}\left(N_{b}\right)$ gauge interaction, we must choose a value of $N_{b}$ and a requisite sector comprised of one or more fermion fields that transform as nonsinglets under both $G=\mathrm{SU}(3)$ and $G_{b}=\mathrm{SU}\left(N_{b}\right)$. For our model we choose a chiral fermion that transforms as an adjoint of $\mathrm{SU}(3)$ and a fundamental representation of $\mathrm{SU}\left(N_{b}\right)$ :

$$
\left(\psi_{j, L}^{i}\right)^{\alpha}: \quad\left(8, N_{b}\right),
$$

where the numbers in parentheses are the dimensions of the representation under $G_{U V}=\mathrm{SU}(3) \otimes \mathrm{SU}\left(N_{b}\right)$, the indices $i, j$ are $\mathrm{SU}(3)$ indices, and $\alpha=1, \ldots, N_{b}$ is an $\mathrm{SU}\left(N_{b}\right)$ index. Because $\left(\psi_{j, L}^{i}\right)^{\alpha}$ transforms according to a self-adjoint representation of $\mathrm{SU}(3)$, it does not contribute any gauge anomaly to the $\mathrm{SU}(3)$ theory. We take $N_{b}=2$, the minimal value, so $G_{b}=\mathrm{SU}(2)_{b}$. As above, we will also use the equivalent notation $\phi_{a, L}, 1 \leq a \leq 8$. The $\left(\psi_{j, L}^{i}\right)^{\alpha}$ form the components of a matrix given by $\sqrt{2} \sum_{a=1}^{8} \psi_{a, L}^{\alpha} T_{a}$.

The $\mathrm{SU}(2)_{b}$ gauge interaction is asymptotically free, with the leading beta function coefficient $b_{1}=14 / 3$ (see Appendix II for notation). The fermion $\left(\psi_{j, L}^{i}\right)^{\alpha}$ amounts to 8 Weyl doublets, or equivalently, 4 Dirac doublets, of $\mathrm{SU}(2)$. Since this number is well below the estimated critical value $N_{f, c r} \simeq 8$ separating the (zero-temperature) phase with confinement and spontaneous chiral symmetry breaking from the chirally symmetric phase [8], we can conclude that the $\mathrm{SU}(2)$ interaction confines and produces bilinear condensates. These occur in the most attractive $\mathrm{SU}(2)_{b}$ channel (MAC), which is $2 \times 2 \rightarrow 1$, with a condensate of the form $\left\langle\epsilon_{\alpha \beta}\left(\psi_{j, L}^{i}\right)^{\alpha T} C\left(\psi_{\ell, L}^{k}\right)^{\beta}\right\rangle$, where here $\epsilon_{\alpha \beta}$ is the antisymmetric tensor density for $\mathrm{SU}(2)_{b}$. The $\epsilon_{\alpha \beta}$ contraction antisymmetrizes the bilinear fermion product, so that in the full Clebsch-Gordan decomposition,

$$
8 \times 8=1_{s}+8_{s}+8_{a}+10_{a}+\overline{10}_{a}+27_{s}
$$


(where the subscripts $s$ and $a$ denote symmetric and antisymmetric combinations), the above condensate must be one of the antisymmetric products, namely $8_{a}$ or $10_{a}$. We next use a vacuum alignment argument [9], according to which the symmetry breaking should preserve as large a subgroup symmetry as possible. Now, relative to the maximal subgroup $\mathrm{SU}(2) \otimes \mathrm{U}(1)$, an octet of $\mathrm{SU}(3)$ has the decomposition

$$
8_{S U(3)}=3_{0}+2_{1}+2_{-1}+1_{0},
$$

where the numbers on the right-hand side are the dimensionalities of the $\mathrm{SU}(2)$ representations and the subscripts are the hypercharges with the Gell-Mann normalization. In contrast, the decuplet has the decomposition

$$
10_{S U(3)}=4_{1}+3_{0}+2_{-1}+1_{-2} .
$$

Of these, only the octet contains a piece that is a singlet under $\mathrm{SU}(2) \otimes \mathrm{U}(1)$. Using a vacuum alignment argument, we therefore can infer that the condensate transforms as the $1_{0}$ piece of the octet of $\mathrm{SU}(3)$ and hence has the form

$$
\left\langle\epsilon_{\alpha \beta}\left(\psi_{j, L}^{i}\right)^{\alpha T} C\left(\psi_{\ell, L}^{j}\right)^{\beta}\right\rangle \propto\left(T_{8}\right)_{\ell}^{i} \Lambda_{b}^{3} .
$$

In the equivalent notation using $\psi_{a, L}^{\alpha}$ with $1 \leq a \leq 8$, the condensate has the form $\left\langle\epsilon_{\alpha \beta} f_{a b 8}\left(\psi_{a, L}\right)^{\alpha T} C\left(\psi_{b, L}\right)^{\beta}\right\rangle$, where the $f_{a b c}$ are the structure constants of the $\mathrm{SU}(3)$ Lie algebra. The nonzero structure constants $f_{a b 8}$ with $a<b$ that enter here are $f_{458}$ and $f_{678}$. The condensate (4.9) dynamically breaks $\mathrm{SU}(3)$ to $\mathrm{SU}(2) \otimes \mathrm{U}(1)$, as in Eq. (4.4). As in the Higgs case, there are four broken generators, namely the $T_{a}$ with $a=4,5,6,7$. The NambuGoldstone modes involving $\psi_{a, L}^{\alpha}, a=4,5,6,7$, contracted on the $\mathrm{SU}(2)_{b}$ indices $\alpha=1,2$ to form $\mathrm{SU}(2)_{b}$ singlets, are absorbed by the corresponding $\mathrm{SU}(3)$ gauge bosons, forming longitudinal polarization states and giving them masses. The remaining $\psi_{a, L}^{\alpha}$ fermions with $a=1,2,3,8$ are bound in $\mathrm{SU}(2)_{b}$-singlet states. Furthermore, of these bound states, the ones with $a=1,2,3$ transform as the adjoint representation of the residual $\mathrm{SU}(2)$ gauge interaction, and, assuming that it confines, they are confined in $\mathrm{SU}(2)$-singlet bound states. This is reminiscent of the situation with the corresponding components of Higgs fields in the situation where one uses a Higgs mechanism for the breaking.

A comment is in order here concerning chiral symmetry in this model. The $\psi_{a, L}^{\alpha}$ fermions have zero Lagrangian masses, and hence, if one turns off the $\mathrm{SU}(3)$ gauge interaction completely, the theory has a (non-anomalous) $\mathrm{SU}(8)$ global chiral symmetry. In general, a full set of bilinear fermion condensates breaks this to $\operatorname{Sp}(8)$. As noted above, the breaking of $\mathrm{SU}(2 k)$ to $\mathrm{Sp}(2 k)$ entails $2 k^{2}-k-1$ broken generators and corresponding massless Nambu-Goldstone bosons. With $k=4$, this means 27 NGBs in the present case. When one turns on the $\mathrm{SU}(3)$ gauge coupling, this explicitly breaks the global SU(8) chiral symmetry, and, moreover, the vacuum alignment argument suggests which condensates form, as we have discussed above.

\section{INDUCED BREAKING OF AN SU $(N)$ SYMMETRY BY ADJOINT FIELDS}

\section{A. General}

In this section we carry out a comparative study of a Higgs mechanism versus dynamical breaking of an $\mathrm{SU}(N)$ gauge symmetry with $N \geq 4$ by fields transforming according to the adjoint representation of this group. Two general types of breaking patterns of the $\mathrm{SU}(N)$ symmetry will be relevant. Both of these involve breaking to a maximal subgroup of $\mathrm{SU}(N)$, with the same rank (dimension of the Cartan subalgebra of mutually commuting generators) as $\mathrm{SU}(N)$, namely $N-1$. However, these subgroups have different orders (numbers of generators). The first of these symmetry-breaking patterns is

$$
\mathrm{SU}(N) \rightarrow \mathrm{SU}(N-1) \otimes \mathrm{U}(1) .
$$

The residual symmetry group has order

$$
o[\mathrm{SU}(N-1) \otimes \mathrm{U}(1)]=(N-1)^{2}
$$

so the symmetry reduction in Eq. (5.1) involves the breaking of

$$
\Delta o=2(N-1)
$$

generators of $\mathrm{SU}(N)$, which is the dimension of the coset

$$
\mathrm{SU}(N) /[\mathrm{SU}(N-1) \otimes \mathrm{U}(1)] .
$$

The second type of symmetry breaking pattern leads to a residual symmetry involving three factor groups. To describe this, it is convenient to deal separately with the cases of even and odd $N$. For even $N=2 k$, a possible symmetry-breaking pattern is

$$
\mathrm{SU}(N) \rightarrow \mathrm{SU}(N / 2) \otimes \mathrm{SU}(N / 2) \otimes \mathrm{U}(1) .
$$

The residual symmetry group has order

$$
o[\mathrm{SU}(N / 2) \otimes \mathrm{SU}(N / 2) \otimes \mathrm{U}(1)]=\frac{N^{2}}{2}-1=2 k^{2}-1,
$$

so that (5.5) involves the breaking of

$$
\Delta o=\frac{N^{2}}{2}=2 k^{2}
$$

generators of $\mathrm{SU}(N)$.

For odd $N=2 k+1$, a possible symmetry-breaking pattern is

$$
\mathrm{SU}(N) \rightarrow \mathrm{SU}((N+1) / 2) \otimes \mathrm{SU}((N-1) / 2) \otimes \mathrm{U}(1) .
$$

The residual symmetry group has order

$$
o[\mathrm{SU}((N+1) / 2) \otimes \mathrm{SU}((N-1) / 2) \otimes \mathrm{U}(1)]
$$




$$
=\frac{N^{2}-1}{2}=2 k(k+1),
$$

so that (5.8) involves the breaking of

$$
\Delta o=\frac{N^{2}-1}{2}=2 k(k+1)
$$

generators of $\mathrm{SU}(N)$. The symmetry breaking patterns (5.5) and (5.8) can be expressed in a unified manner as

$$
\mathrm{SU}(N) \rightarrow \mathrm{SU}(N-\ell) \otimes \mathrm{SU}(\ell) \otimes \mathrm{U}(1),
$$

where $\ell=[N / 2]_{i p}$ and $[\nu]_{i p}$ denotes the integral part of the real number $\nu$.
As we will discuss further below in the context of dynamical symmetry breaking, a vacuum alignment argument prefers a symmetry-breaking pattern that yields the largest residual symmetry. The size of the subgroup that constitutes the residual symmetry can be characterized by its rank and order. All of the patterns above satisfy the condition that the rank of the residual symmetry group should be maximal, i.e., the same as that of $\mathrm{SU}(N)$, namely $N-1$. Concerning the differences in the orders of the various possible subgroups resulting from the symmetry breaking of $\mathrm{SU}(N)$, we calculate, for even $N=2 k$, the difference

$$
o[\mathrm{SU}(N-1) \otimes \mathrm{U}(1)]-o[\mathrm{SU}(N / 2) \otimes \mathrm{SU}(N / 2) \otimes \mathrm{U}(1)]=\frac{(N-2)^{2}}{2}=2(k-1)^{2} .
$$

This difference is positive semidefinite, and positive-definite for $k \geq 2$, i.e., $N \geq 4$. For odd $N=2 k+1$,

$$
o[\mathrm{SU}(N-1) \otimes \mathrm{U}(1)]-o[\mathrm{SU}((N+1) / 2) \otimes \mathrm{SU}((N-1) / 2) \otimes \mathrm{U}(1)]=\frac{(N-1)(N-3)}{2}=2 k(k-1) .
$$

This difference is also positive semidefinite, and positivedefinite for $k \geq 2$, i.e., $N \geq 5$. Hence, as these calculations show, a vacuum alignment argument prefers the breaking pattern (5.1) for both even and odd $N \geq 4$. The special case $N=3$ has been analyzed above, and leads to breaking of the $\mathrm{SU}(3)$ group to $\mathrm{SU}(2) \times \mathrm{U}(1)$, which is also of the form (5.1) with $N=3$.

There are other symmetry-breaking patterns that could, a priori, occur. $\mathrm{SU}(N)$ could, in principle, break to a non-maximal subgroup, i.e., a subgroup with rank smaller than the rank of $\mathrm{SU}(N)$, namely $N-1$. For example, in principle $\mathrm{SU}(3)$ could, a priori break to $\mathrm{U}(1), \mathrm{SU}(4)$ could break to $\mathrm{SU}(2) \otimes \mathrm{U}(1)$, and so forth. However, in the context of the Higgs mechanism, these symmetry-breaking patterns do not occur as minima of the Higgs potential, and in the dynamical symmetry breaking context, they are disfavored by vacuum alignment arguments.

\section{B. SU( $N)$ Breaking with an Adjoint Higgs Field}

First, we discuss the mechanism for breaking an $\mathrm{SU}(N)$ gauge symmetry with a Higgs field $\Phi$ in the adjoint representation [14]. The components of the Higgs field are denoted $\Phi_{j}^{i}$. We impose a $\Phi \rightarrow-\Phi$ symmetry. Then the Higgs potential has the general form

$$
V=\frac{\mu^{2}}{2} \operatorname{Tr}\left(\Phi^{2}\right)+\frac{\lambda_{1}}{4}\left[\operatorname{Tr}\left(\Phi^{2}\right)\right]^{2}+\frac{\lambda_{2}}{4} \operatorname{Tr}\left(\Phi^{4}\right),
$$

where we take $\mu^{2}<0$ to produce the symmetry breaking. Since $\Phi$ is a hermitian matrix, it can be diagonalized by a unitary transformation. It follows that one can write

$$
\Phi_{j}^{i}=\delta_{j}^{i} \phi_{j} \quad(\text { no sum on } j),
$$

for $1 \leq i, j \leq N$. Substituting Eq. (5.15) into Eq. (5.14) gives

$$
V=\frac{\mu^{2}}{2} \sum_{i=j}^{N} \phi_{j}^{2}+\frac{\lambda_{1}}{4}\left(\sum_{j=1}^{N} \phi_{j}^{2}\right)^{2}+\frac{\lambda_{2}}{4} \sum_{j=1}^{N} \phi_{j}^{4} .
$$

Since $\operatorname{Tr}(\Phi)=0$, the $\phi_{j}$ satisfy the condition

$$
\sum_{j=1}^{N} \phi_{j}=0
$$

Hence, $\phi$ only involves $N-1$ independent fields, and $V$ only depends on $N-1$ of the components $\phi_{j}$, which we take to be $\phi_{j}$ with $j=1, \ldots, N-1$. Now

$$
\left[\operatorname{Tr}\left(\Phi^{2}\right)\right]^{2} \geq \operatorname{Tr}\left(\Phi^{4}\right)
$$

as can be seen from the explicit expression for the difference,

$$
\begin{aligned}
& {\left[\operatorname{Tr}\left(\Phi^{2}\right)\right]^{2}-\operatorname{Tr}\left(\Phi^{4}\right)=} \\
& 2\left[\sum_{1 \leq i<j \leq N-1} \phi_{i}^{2} \phi_{j}^{2}+\left(\sum_{i=1}^{N-1} \phi_{i}^{2}\right)\left(\sum_{j=1}^{N-1} \phi_{j}\right)^{2}\right]
\end{aligned}
$$




$$
\geq 0
$$

As noted above, if $N$ is equal to 2 or 3 , then $\left[\operatorname{Tr}\left(\Phi^{2}\right)\right]^{2}=$ $2 \operatorname{Tr}\left(\Phi^{4}\right)$, so that there is only one independent quartic term, and its coefficient, $(1 / 4)\left[\lambda_{1}+\left(\lambda_{2} / 2\right)\right]$, must be positive. For $N \geq 4$, the two quartic terms are independent, and the condition that $V$ be bounded from below requires that $\lambda_{1}>0$.

For $\lambda_{2}>0$, it is again convenient to consider the cases of even $N=2 k$ and odd $N=2 k+1$ separately. For $\lambda_{2}>0$ and even $N=2 k, V$ is minimized if the $\mathrm{VEV}$ of $\Phi$ has the form given by

$$
\left\langle\phi_{i}\right\rangle=\frac{v}{\sqrt{2 N}} \times\left\{\begin{array}{ll}
1 & \text { for } 1 \leq i \leq k \\
-1 & \text { for } k+1 \leq i \leq 2 k
\end{array} .\right.
$$

The normalization of $v$ in Eq. (5.20) and in the equations below is determined by the definition $\operatorname{Tr}\left(\Phi^{2}\right)=$ $(1 / 2) v^{2}$, analogous to the usual normalization condition $\operatorname{Tr}\left(T_{a} T_{b}\right)=(1 / 2) \delta_{a b}$ for the generators of $\mathrm{SU}(N)$. At this minimum, one finds

$$
v^{2}=\frac{-2 \mu^{2}}{\left[\lambda_{1}+\frac{\lambda_{2}}{N}\right]} .
$$

The VEV (5.20) breaks SU( $2 k)$ according to (5.5). The value of the potential at the minimum is

$$
V_{\min }=\frac{-\mu^{4}}{4\left[\lambda_{1}+\frac{\lambda_{2}}{N}\right]}
$$

For $\lambda_{2}>0$ and odd $N=2 k+1$ with $k \geq 2, V$ is minimized if the $\mathrm{VEV}$ of $\Phi$ has the form

$$
\begin{aligned}
\left\langle\phi_{i}\right\rangle & =v\left[\frac{k}{2(k+1)(2 k+1)}\right]^{1 / 2} \times \\
& \times\left\{\begin{array}{ll}
1 & \text { for } 1 \leq i \leq k+1 \\
-\frac{k+1}{k} & \text { for } k+2 \leq i \leq 2 k+1
\end{array} .\right.
\end{aligned}
$$

(The special case $k=1$, i.e., $N=3$, was dealt with above.) The minimization condition determines $v$ according to

$$
v^{2}=\frac{-2 \mu^{2}}{\left[\lambda_{1}+\left(\frac{N^{2}+3}{N(N+1)(N-1)}\right) \lambda_{2}\right]} .
$$

This VEV (5.23) breaks SU( $2 k+1)$ according to (5.8). The value of the potential at the minimum is

$$
V_{\text {min }}=\frac{-\mu^{4}}{4\left[\lambda_{1}+\left(\frac{N^{2}+3}{N(N+1)(N-1)}\right) \lambda_{2}\right]}
$$

It is possible for $\lambda_{2}$ to have a restricted range of negative values [15],

$$
-\left(\frac{N(N-1)}{N^{2}-3 N+3}\right) \lambda_{1}<\lambda_{2}<0 .
$$

For $\lambda_{2}$ in this range, $V$ is minimized if $\Phi$ has the $\mathrm{VEV}$

$$
\left\langle\phi_{i}\right\rangle=\frac{v}{\sqrt{2 N(N-1)}} \times \begin{cases}1 & \text { for } 1 \leq i \leq N-1 \\ -(N-1) & \text { for } i=N\end{cases}
$$

where

$$
v^{2}=\frac{-2 \mu^{2}}{\left[\lambda_{1}+\left(\frac{N^{2}-3 N+3}{N(N-1)}\right) \lambda_{2}\right]} .
$$

The VEV (5.27) breaks $\mathrm{SU}(N)$ according to Eq. (5.1). The value of $V$ at this minimum is

$$
V_{\text {min }}=\frac{-\mu^{4}}{4\left[\lambda_{1}+\left(\frac{N^{2}-3 N+3}{N(N-1)}\right) \lambda_{2}\right]}
$$

Note that all three of the minimal values (5.22), (5.25), and (5.29) have the form

$$
V_{\min }=\frac{\mu^{2} v^{2}}{8}
$$

for the respective three values of $v^{2}$. The lower limit on the allowed negative range of $\lambda_{2}$ in Eq. (5.26) is evident from Eq. (5.29), since in this equation $V_{\min } \rightarrow-\infty$ as $\lambda_{2}$ approaches this lower limit from above. The fact that $\lambda_{2}=0$ is the boundary between the two types of minima is evident from the difference between the values of the minima for even $N$,

$$
V_{\min , \lambda_{2}>0}-V_{\min , \lambda_{2}<0}==\frac{-(N-2)^{2} \lambda_{2} \mu^{4}}{4\left[\lambda_{1}+\frac{\lambda_{2}}{N}\right]\left[N(N-1) \lambda_{1}+\left(N^{2}-3 N+3\right) \lambda_{2}\right]}
$$


and for odd $N$,

$$
V_{\min , \lambda_{2}>0}-V_{\min , \lambda_{2}<0}=\frac{-N^{3}(N-1)(N-3) \lambda_{2} \mu^{4}}{4\left[N\left(N^{2}-1\right) \lambda_{1}+\left(N^{2}+3\right) \lambda_{2}\right]\left[N(N-1) \lambda_{1}+\left(N^{2}-3 N+3\right) \lambda_{2}\right]} .
$$

Both of these differences are proportional to $\lambda_{2}$, explicitly showing the switch in global minimum as $\lambda_{2}$ reverses sign.

The reason for the residual $\mathrm{U}(1)$ invariance in these symmetry-breaking patterns obtained with a Higgs field $\Phi$ transforming according to the adjoint representation of $\mathrm{SU}(N)$ is that since $\Phi$ can be diagonalized, as noted above, its VEV can be expressed as a linear combination of coefficients multipled by the $N-1$ diagonal Cartan generators of $\mathrm{SU}(N)$. Indeed, without loss of generality, one can define axes in $\mathrm{SU}(N)$ space so that it points entirely along one such Cartan generator, which can be denoted as $T_{C}$. Then $\exp \left(i \theta T_{C}\right)$ commutes with $\langle\Phi\rangle$, yielding the $\mathrm{U}(1)$ invariance.

From the formulas for $\Delta o$, the number of broken generators for the various symmetry-breaking patterns, one can immediately infer the number of gauge bosons of $\mathrm{SU}(N)$ that become massive. Thus, for $\lambda_{2}>0$ and even $N=2 k$, the symmetry breaking (5.5) involves the breaking of $N^{2} / 2=2 k^{2}$ generators, so that of the $N^{2}-1$ (real) components of $\Phi, N^{2} / 2$ are absorbed to become the longitudinal components of the gauge bosons corresponding to these broken generators, which pick up masses $\propto g v$. The remaining $N^{2} / 2-1=2 k^{2}-1$ real components of $\Phi$ are physical Higgs bosons. For $\lambda_{2}>0$ and odd $N=2 k+1$, the symmetry breaking (5.8) involves the breaking of $\left(N^{2}-1\right) / 2=2 k(k+1)$ generators, so that of the $N^{2}-1$ (real) components of $\Phi,\left(N^{2}-1\right) / 2$ are absorbed to become the longitudinal components of the gauge bosons corresponding to these broken generators. The remaining $\left(N^{2}-1\right) / 2=2 k(k+1)$ real components of $\Phi$ are physical Higgs bosons. For $\lambda_{2}<0$, the symmetry breaking (5.1) involves the breaking of $2(N-1)$ generators, and an equal number of Nambu-Goldstone bosons, which are absorbed to become the longitudinal components of the gauge bosons in the coset (5.4). The remaining $(N-1)^{2}$ real components of $\Phi$ are physical Higgs bosons.

\section{Dynamical Mechanism for $\mathrm{SU}(N)$ Breaking by an Adjoint Field}

For the analysis of dynamical symmetry breaking of $\mathrm{SU}(N)$ by an adjoint field, we analyze a model of the form of Eq. (1.1), in which

$$
G=\mathrm{SU}(N), \quad G_{b}=\mathrm{SU}\left(N_{b}\right) .
$$

For the fermions that transform under both $\mathrm{SU}(N)$ and $G_{b}$ we use

$$
\left(\psi_{j}^{i}\right)_{L}^{\alpha}: \quad\left(N^{2}-1, N_{b}\right)
$$

where here and below, $\alpha$ is the $G_{b}$ gauge index. Thus, we assign each of the $N^{2}-1$ components of $\left(\psi_{j}^{i}\right)_{L}^{\alpha}$ to transform according to the fundamental representation of $\mathrm{SU}\left(N_{b}\right)$. The numbers in parentheses in Eq. (5.34) are the dimensions of the representations with respect to the factor groups in Eq. (5.33). The $N_{b}$ copies of fermions in the adjoint representation of $\mathrm{SU}(N)$ contribute zero gauge anomaly to $\mathrm{SU}(N)$. As stated earlier, these and the other fermions that we include are taken to have zero Lagrangian masses since mass terms would violate the full $G_{U V}$ symmetry, which is chiral.

The choice of the rest of the $G_{b}$-nonsinglet fermions in the model depends on the value of $N_{b}$. We first consider the possibility that $N_{b}=2$. Now, $N$ is even $\Longleftrightarrow$ $N^{2}-1$ is odd. The $\mathrm{SU}(2)_{b}$ theory must have an even number of chiral doublet fermions in order to avoid a global anomaly, so if $N$ is odd, the $N^{2}-1\left(\psi_{j}^{i}\right)_{L}^{\alpha}$ form an acceptable $\mathrm{SU}(2)_{b}$ fermion sector by themselves, while if $N$ is even, then we obtain an acceptable fermion sector by adding an odd number of additional $\mathrm{SU}(2)_{b}$ doublets. We shall choose this odd number to be the minimal value, namely, one, with the fermion

$$
\omega_{L}^{\alpha} \quad \text { included for even } N .
$$

For these two cases, the $\mathrm{SU}(2)_{b}$ beta function has as its leading coefficient

$$
b_{1}= \begin{cases}\frac{1}{3}\left(23-N^{2}\right) & \text { for } N \text { odd } \\ \frac{1}{3}\left(22-N^{2}\right) & \text { for } N \text { even }\end{cases}
$$

The requirement that the $\mathrm{SU}(2)_{b}$ theory be asymptotically free is thus that $N<\sqrt{23}$ for odd $N$ and $N<\sqrt{22}$ for even $N$. These amount to the possibilities $N=3$ for odd $N$ and $N=2,4$ for even $N$. We have already dealt with the case $N=3$ above, so here we focus on the case $N=4$. As discussed in the introduction, these are necessary but not sufficient conditions; we also must require that the fermion content of the $\mathrm{SU}(2)_{b}$ theory is sufficiently small that as the reference energy scale $\mu$ decreases, the coupling $\alpha_{b}(\mu)$ will increase sufficiently so that the $\mathrm{SU}(2)_{b}$ gauge interaction will produce bilinear fermion condensates instead of evolving in a chirally symmetric manner into the infrared. For SU(2), the critical number of Dirac fermions, $N_{f, c r}$, below which this condensation will occur is estimated to be $N_{f, c r} \simeq 8$ [8]. Because SU(2) has only (pseudo)real representations, we can rewrite the theory with a given number of chiral Weyl doublets as a theory with half this number of Dirac dou- 
blets. For $N=4$, we would have $N^{2}=16$ chiral doublets, or eight Dirac doublets, which is marginal. Assuming that the $\mathrm{SU}(2)_{b}$ sector does, indeed, produce bilinear fermion condensates, these would occur in the most attractive channel, which is $2 \times 2 \rightarrow 1$ in $\mathrm{SU}(2)_{b}$. These would have either the form

$$
\left\langle\epsilon_{\alpha \beta}\left[\psi_{a, L}^{\alpha T} C \psi_{b, L}^{\beta}\right]_{a s}\right\rangle
$$

or the form

$$
\left\langle\epsilon_{\alpha \beta} \psi_{a, L}^{\alpha T} C \omega_{L}^{\beta}\right\rangle
$$

where in Eq. (5.37) the symbol [... $]_{\text {as }}$ means an antisymmetric SU(4) combination of the two adjoint fermion fields. In both cases, the condensate thus transforms as an adjoint of SU(4). A vacuum alignment argument implies that the condensates form in such a way as to preserve the largest subgroup in $\mathrm{SU}(4)$. The order of the subgroup $\mathrm{SU}(3) \otimes \mathrm{U}(1)$ is 9 , which is greater than the order of the subgroup $\mathrm{SU}(2) \otimes \mathrm{SU}(2) \otimes \mathrm{U}(1)$, which is 7. Hence, from a vacuum alignment argument, one may infer that the condensate is proportional to the $\mathrm{SU}(4)$ generator $T_{15}=(2 \sqrt{6})^{-1} \operatorname{diag}(1,1,1,-3)$, leading to the $N=4$ special case of the symmetry-pattern pattern (5.1).

We next consider possible values $N_{b} \geq 3$ for the gauge group symmetry $\mathrm{SU}\left(N_{b}\right)$ responsible for the dynamical breaking of $\mathrm{SU}(N)$. In this case, for the rest of the $G_{b^{-}}$ nonsinglet fermions we choose

$$
\omega_{p, L}^{\alpha}: \quad\left(N^{2}-1\right)\left(1, \bar{N}_{b}\right)
$$

where the notation $\bar{N}_{b}$ means the conjugate fundamental representation and here the copy number takes on the values $1 \leq p \leq N^{2}-1$. This ensures that the $\operatorname{SU}\left(N_{b}\right)$ theory has zero gauge anomaly. With the fermions (5.34) and (5.39) (and with the $\mathrm{SU}(N)$ interaction taken as negligibly weak), the $\mathrm{SU}\left(N_{b}\right)$ theory is vectorlike. This is in accord with one of the conditions that we imposed above, which guarantees that the $G_{b}$ symmetry does not selfbreak when it becomes strongly coupled. Expressed in manifestly vectorial form, it has $N^{2}-1$ Dirac fermions transforming according to the fundamental representation of $\mathrm{SU}\left(N_{b}\right)$.

The beta function for the $\mathrm{SU}\left(N_{b}\right)$ coupling has leading coefficient

$$
\left(b_{1}\right)_{S U\left(N_{b}\right)}=\frac{1}{3}\left[11 N_{b}-2\left(N^{2}-1\right)\right] .
$$

The requirement that the $\mathrm{SU}\left(N_{b}\right)$ theory be asymptotically free is thus

$$
N_{b}>\frac{2\left(N^{2}-1\right)}{11} .
$$

As noted above, this is a necessary, but not sufficient, condition for the $\mathrm{SU}\left(N_{b}\right)$ theory to produce the requisite condensates. We must also require that, for a given value of $N_{b}$, the fermion content of the $\mathrm{SU}\left(N_{b}\right)$ sector must be small enough so that as the theory evolves down in energy scale, it produces condensates instead of evolving into the infrared in a chirally symmetric (conformal) manner. For a vectorial asymptotically free $\mathrm{SU}(N)$ gauge theory with $N_{f}$ copies of Dirac fermions (with zero Lagrangian masses) in the fundamental representation, if $N_{f}$ is smaller than a critical value, $N_{f, c r}$, then as the reference scale decreases from large values, the coupling will eventually grow large enough to form condensates which generically break the global chiral symmetry. In contrast, if $N_{f}>N_{f, c r}$ then the theory will evolve from the ultraviolet to the infared without any spontaneous chiral symmetry breaking, yielding conformal behavior. A combined analysis of the beta function and solutions of the Dyson-Schwinger equation for the fermion propagator in the approximation of one-gluon exchange yields the result [8]

$$
N_{f, c r}=\frac{2 N_{b}\left(50 N_{b}^{2}-33\right)}{5\left(5 N_{b}^{2}-3\right)} .
$$

Although the Dyson-Schwinger analysis does not directly incorporate either effects of confinement or instantons, it has been shown that these two effects affect $N_{f, c r}$ in opposite ways, so that neglecting both of them can still yield a reasonably accurate result [16]. Recent lattice simulations of $\mathrm{SU}(3)$ gauge theory with variable numbers $N_{f}$ of light fermions in the fundamental representation are (taking account of theoretical uncertainties in both Eq. (5.42) and the lattice work) broadly consistent with Eq. (5.42) [17]. Although this does not test the prediction for $N_{b} \neq 3$, it makes it plausible that this prediction could also be reasonably accurate. For these values of $N_{b}$, Eq. (5.42) rapidly approaches the asymptotic large- $N_{b}$ form $N_{f, c r} \simeq 4 N_{b}$. We thus require that $N_{b}$ is sufficiently large that the $\mathrm{SU}\left(N_{b}\right)$ theory with its $N_{f}=N^{2}-1$ Dirac fermions will exhibit spontaneous chiral symmetry breaking and confinement instead of evolving down in energy in a chirally symmetric non-Abelian Coulomb (conformal) phase. Using the prediction of Eq. (5.42), we thus obtain the lower bound $N_{f, c r} \simeq 4 N_{b}>N^{2}-1$, i.e.,

$$
N_{b}>\frac{\left(N^{2}-1\right)}{4} \text {. }
$$

With the fermion content as specified via Eqs. (5.34) and (5.39), and in the approximation that one turns off the $\mathrm{SU}(N)$ gauge interaction, the $\mathrm{SU}\left(N_{b}\right)$ sector has a classical global symmetry of the form $\mathrm{U}\left(N^{2}-1\right)_{\psi} \otimes$ $\mathrm{U}\left(N^{2}-1\right)_{\omega}$, or equivalently, $\mathrm{SU}\left(N^{2}-1\right)_{\psi} \otimes \mathrm{SU}\left(N^{2}-1\right)_{\omega} \otimes$ $\mathrm{U}(1)_{\psi} \otimes \mathrm{U}(1)_{\omega}$, where the subscripts indicate which fields are involved in the respective symmetry transformations. Both the $\mathrm{U}(1)_{\psi}$ and $\mathrm{U}(1)_{\omega}$ are broken by $\mathrm{SU}\left(N_{b}\right)$ instantons, but the linear combination corresponding to the difference of the currents for the $\psi$ and $\omega$ fields is conserved in the presence of instantons. We will denote this symmetry as $\mathrm{U}(1)^{\prime}$. The actual (non-anomalous) global symmetry of the $G_{b}$ theory at the high scale is thus

$$
\mathrm{SU}\left(N^{2}-1\right)_{\psi} \otimes \mathrm{SU}\left(N^{2}-1\right)_{\omega} \otimes \mathrm{U}(1)^{\prime} .
$$


We comment on this further below.

Now we turn on the $\mathrm{SU}(N)$ gauge interaction. This explicitly breaks the above global chiral symmetry. However, just as the breaking of chiral $\mathrm{SU}(2)_{L} \otimes \mathrm{SU}(2)_{R}$ symmetry in QCD by electroweak interactions is weak, so also here this breaking is weak, since $\alpha_{G}$ is small at the scale $\Lambda_{b}$. We can fix the initial value of $\alpha_{b}(\mu)$ at a high value of $\mu$ so that as $\mu$ decreases to the scale $\Lambda_{b}$, this coupling grows sufficiently large to produce bilinear fermion condensates. These condensates will occur in the most attractive channel, which, for the above fermion content, is $N_{b} \times \bar{N}_{b} \rightarrow 1$. In general, these condensates would be of the form $\left\langle\psi_{a, L}^{\alpha T} C \omega_{p, \alpha, L}\right\rangle$. A vacuum alignment argument implies that these condensates will form in a manner such as to preserve the largest residual gauge symmetry. We regard this implication as very plausible, but add the obvious caveat that one must remember the theoretical uncertainties that are present in such a strongly coupled theory. Combining this implication from the vacuum alignment argument with our discussion above, we infer that the symmetry-breaking pattern is that $\mathrm{SU}(N)$ breaks to the maximal subgroup $\mathrm{SU}(N-1) \otimes \mathrm{U}(1)$ as in Eq. (5.1), so that the condensate would have the form

$$
\left\langle\psi_{a, L}^{\alpha T} C \omega_{p, \alpha, L}\right\rangle \quad \text { with } a=N^{2}-1
$$

That is, it would transform like the last of the generators in the Cartan algebra of $\mathrm{SU}(N)$,

$$
\begin{array}{ll}
\left(T_{a=N^{2}-1}\right)_{j}^{i}= & \\
\frac{1}{\sqrt{2 N(N-1)}} \delta_{j}^{i} \begin{cases}1 & \text { for } 1 \leq i \leq N-1 \\
-(N-1) & \text { for } i=N\end{cases}
\end{array}
$$

Here we emphasize an important contrast between this dynamical symmetry breaking mechanism and the Higgs mechanism. In our introductory discussion above, we have already noted a number of the differences between the Higgs mechanism and a dynamical mechanism for breaking a gauge symmetry. Among other differences, for example, the Higgs mechanism leads to the appearance of at least one physical pointlike Higgs field, whereas a dynamical mechanism does not yield such a particle (although it may yield composite $J=0$ bound states). Furthermore, if one uses a Higgs mechanism to break $\mathrm{SU}(N)$, then by appropriate choices of the parameters, one can guarantee that the minimim of the potential occurs for a Higgs VEV of the form (5.20) or (5.23), so that the symmetry breaking is of the type (5.5) or (5.8), rather than (5.1). However, in the dynamical approach to $\mathrm{SU}(N)$ breaking, once one specifies the gauge and fermion content, there are no free parameters, and the theory is, in principle, completely predictive. Although the dynamical symmetry-breaking mechanism involves a strongly coupled gauge sector, one can use most attractive channel criteria and vacuum alignment arguments to make a plausible inference about what form the bilinear fermion condensate will take, namely, as discussed above, the form that preserves the largest residual symmetry, $\mathrm{SU}(N-1) \otimes \mathrm{U}(1)$. These MAC and vacuum alignment properties would be manifest if one were to explicitly calculate the effective potential for the composite operator represented by the condensate, along the lines of Ref. [18]. In this context, one may recall that the Higgs potential was partially motivated by the original GinzburgLandau free energy functional in phenomenological models of superconductivity, and retrospectively, from the perspective of the Bardeen-Cooper-Schrieffer theory and the Cooper pair condensate, one may view the GinzburgLandau free energy functional as an approximate way to represent the physics of this Cooper pair condensate. This is, of course, not a precise isomorphism, but only a partial correspondence. As recalled above, there are important differences between a Higgs and dynamical mechanism for breaking a gauge symmetry. To the extent that one may regard a Higgs potential as embodying some of the same physics as an effective potential for a composite operator represented by bilinear fermion condensate(s), one may observe that the pattern of symmetry breaking inferred from the dynamical approach makes definite predictions for the coefficients in the corresponding Higgs potential. First, because the Lagrangian in the dynamical model is invariant under the separate global transformations $\psi_{a, L}^{\alpha} \rightarrow-\psi_{a, L}^{\alpha}$ and $\omega_{p, L}^{\alpha} \rightarrow-\omega_{p, L}^{\alpha}$, it follows that an analogous effective potential for the condensate (5.45) should not contain odd powers of this condensate. Our dynamical model for the symmetry breaking of an $\mathrm{SU}(N)$ gauge theory using fermions transforming as an adjoint representation of $\mathrm{SU}(N)$ then predicts that in a corresponding Higgs approach, in order to obtain the same pattern of symmetry breaking, the coefficients of the Higgs potential should have the following properties: (i) $\mu^{2}<0$, for symmetry breaking; (ii) $\lambda_{2}<0$, yielding the specific symmetry-breaking pattern (5.1); and the stability properties that (iii) $\lambda_{1}>0$ and (iv) $\lambda_{2}$ satisfies the lower bound in Eq. (5.26).

With the symmetry-breaking pattern as given by (5.1), there are then $2(N-1)$ broken generators of $\mathrm{SU}(N)$, and Nambu-Goldstone modes formed from the fermion condensates are absorbed by the gauge bosons corresponding to these broken generators, forming the longitudinal components of the resultant massive vector bosons. These masses are of order $g \Lambda_{b}$. This is reminiscent of the process whereby Nambu-Goldstone modes in the technicolor mechanism for electroweak symmetry breaking are absorbed to give the $W^{ \pm}$and $Z$ bosons their masses. The $\mathrm{SU}\left(N_{b}\right)$-nonsinglet fermions involved in the condensate (5.45) gain dynamical masses of order $\Lambda_{b}$ and are integrated out of the low-energy effective theory that is operative at scales $\mu$ below $\Lambda_{b}$. Since, by construction, the $\mathrm{SU}\left(N_{b}\right)$ theory confines, the spectrum of the $\mathrm{SU}\left(N_{b}\right)$ theory includes a set of $\mathrm{SU}\left(N_{b}\right)$-singlet mesons, baryons, and glueballs that form at the scale $\Lambda_{b}$. 


\section{REMARKS ON OTHER DIRECTIONS OF STUDY}

We comment here on some other related directions of study that could be interesting to pursue. One could construct models with dynamical symmetry breaking of other gauge symmetries and compare results with those obtained via Higgs scenarios. An example of this would be models with extended electroweak gauge groups such as $G=\mathrm{SU}(3)_{c} \otimes \mathrm{SU}(2)_{L} \otimes \mathrm{SU}(2)_{R} \otimes \mathrm{U}(1)_{B-L}$ and $G=\mathrm{SU}(4) \otimes \mathrm{SU}(2)_{L} \otimes \mathrm{SU}(2)_{R}$, for which dynamical mechanisms were presented in Ref. [19]. In a more abstract direction, one could consider groups such as $G=\mathrm{SO}(N)$. One could also study the breaking of $\mathrm{SU}(N)$ by fields transforming according to representations other than the fundamental and adjoint, such as the rank-2 symmetric and antisymmetric tensor representations.

One could also study situations in which the $G$ gauge interaction is not weakly coupled at the scale $\Lambda_{b}$ where the $G_{b}$ interaction becomes strongly coupled, so that there is generically a combination of self-breaking of $G$ and induced breaking of $G$ by $G_{b}$. Indeed, in reasonably ultra-violet-complete extended technicolor (ETC) theories, the sequential breaking of the ETC gauge symmetry down to the residual exact technicolor symmetry typically involves both self-breaking of ETC, which is a strongly coupled, chiral gauge symmetry, and induced breaking by an auxiliary gauge interaction called hypercolor in [20]. A similar statement applies to ultraviolet completions of topcolor-assisted technicolor models that include the necessary additional gauge interactions to produce the required symmetry breakings [3, 21].

Although our study is primarily intended as a comparison of gauge symmetry breaking by dynamical and Higgs mechanisms in a general field theoretic context, it is appropriate to address the question of possible dynamical symmetry breaking of a grand unified symmetry. We recall that there has long been interest in grand unified theories (GUTs) which embed the three factor groups of $G_{S M}$ in a single group, since this would unify quarks and leptons, predict the ratios of the three SM gauge couplings, and quantize electric charge [22]- 224]. Much work on GUTs has been done in a supersymmetric context, since supersymmetry remedies the gauge hierarchy problem of the Standard Model and since the MSSM naturally yields gauge coupling unification. There have also been studies of the question of whether some type of grand unification could feasibly be achieved in a theoretical context involving dynamical electroweak symmetry breaking [25]. It is natural to ask whether one could use induced dynamical breaking of a GUT gauge symmetry such as $\mathrm{SU}(5)$ or $\mathrm{SO}(10)$, which is weakly coupled at the GUT scale, $M_{G U T}$, using a (vectorial non-Abelian, asymptotically free) $G_{b}$ gauge interaction that becomes strongly coupled at this scale. One could, of course, argue that such an approach differs from the original purpose of the grand unification, which was to obtain an ultraviolet-scale theory with only a single gauge group and gauge coupling. Indeed, such an induced GUT symmetry breaking scenario appears problematic, since in order to produce the requisite bilinear fermion condensates, the $G_{b}$ interaction would necessarily have to confine, and this would generically lead to stable $G_{b}$-singlet baryons with masses of order $M_{G U T}$. With plausible estimates for the relevant reaction cross sections, one finds that these $G_{b}$-singlet baryons would contribute far too much to the dark matter in the universe [26]. Interestingly, even if a dynamical approach to breaking a GUT symmetry were not excluded by its production of excessive dark matter, it would predict that a GUT group such as $\mathrm{SU}(5)$ would preferentially break to $\mathrm{SU}(4) \otimes \mathrm{U}(1)$ rather than the $\mathrm{SM}$ group, $\mathrm{SU}(3)_{c} \otimes \mathrm{SU}(2)_{L} \otimes \mathrm{U}(1)_{Y}$. In the conventional SU(5) GUT, the latter breaking to $G_{S M}$ is obtained by a Higgs mechanism with a Higgs field transforming as the adjoint representation [22]. Modern GUT theories also make use of string-inspired mechanisms for the GUT gauge symmetry breaking, including higherdimension operators and Wilson lines 24].

\section{CONCLUSIONS}

In conclusion, in this paper we have constructed and analyzed theories with a gauge symmetry in the ultraviolet of the form $G \otimes G_{b}$, in which the vectorial, asymptotically free $G_{b}$ gauge interaction becomes strongly coupled at a scale where the $G$ interaction is weakly coupled and produces bilinear fermion condensates that dynamically break the $G$ symmetry. We have compared the results to those obtained with a Higgs mechanism. There are many interesting contrasting properties of these two approaches to breaking a gauge symmetry. The Higgs mechanism is perturbative, and one has the freedom, by appropriate choices of parameters in the Higgs potential, to determine whether and, in general, how the symmetry breaks. In contrast, the dynamical approach is arguably more predictive, in the sense that, provided that one has chosen the gauge and field content of the $G_{b}$ sector appropriately, there are no free parameters to vary; the $G_{b}$ gauge interaction will confine and produce fermion condensates that break the $G$ symmetry. Most attractive channel and vacuum alignment arguments provide a plausible guide to enable one to infer which channel(s) have fermion condensation, and what the form of this condensation is, thereby predicting the resultant pattern of symmetry breaking. In the dynamical models that we have constructed, we produce this breaking by introducing fermions that are nonsinglets under both $G$ and $G_{b}$. In the course of our analysis, we have discussed how the gauge symmetry $G$ can be broken not just for the case where it is chiral (as in electroweak symmetry breaking), but also for the case where it is vectorial. We have compared Higgs and dynamical mechanisms for breaking SU(3) via a Higgs field or condensate transforming according to the fundamental or adjoint representation. We have also carried such an analogous study for $\mathrm{SU}(N)$ with $N \geq 4$. Our present 
study helps to elucidate the differences between the Higgs and dynamical mechanisms for breaking a gauge symmetry. We believe such theoretical studies are useful since it is still an open question what mechanism is responsible for breaking electroweak gauge symmetry or a grand unified symmetry.

Acknowledgments: This research was partially supported by the grant NSF-PHY-06-53342.

\section{APPENDIX I}

Here we define some notation used in the text. For a gauge group $G_{j}$ we denote the running gauge coupling as $g_{j}(\mu)$, where $\mu$ is the Euclidean reference momentum, and we denote $\alpha_{j}(\mu)=g_{j}(\mu)^{2} /(4 \pi)$. The beta function is $\beta_{G_{j}}=d g_{j} / d t$, where $d t=d \ln \mu$. We write

$$
\frac{d \alpha_{j}}{d t}=-\frac{\alpha_{j}^{2}}{2 \pi}\left[b_{1}+\frac{b_{2} \alpha_{j}}{4 \pi}+O\left(\alpha_{j}^{3}\right)\right]
$$

where the first two coefficients, $b_{1}$ and $b_{2}$, are schemeindependent. For a representation $R$ of a Lie group $G$, the quadratic Casimir invariant $C_{2}(R)$ is defined by $\sum_{a=1}^{\operatorname{order}(G)} \sum_{j=1}^{\operatorname{dim}(R)}\left(T_{a}\right)_{i j}\left(T_{a}\right)_{j k}=C_{2}(R) \delta_{i k}$.
[1] S. Weinberg, Phys. Rev. D 19, 1277 (1979); L. Susskind, Phys. Rev. D 20, 2619 (1979)

[2] C. Quigg and R. Shrock, Phys. Rev. D 79, 096002 (2009).

[3] C. T. Hill and E. H. Simmons, Phys. Rep. 381, 235 (2003); Workshop on Dynamical Electroweak Symmetry Breaking, Southern Denmark Univ. 2008 (http://hep.sdu.dk/dewsb); R. S. Chivukula, M. Narain, and J. Womersley, in Particle Data Group, J. Phys. G 37 1340, (2010); F. Sannino, Acta Phys. Polon. B 40, 3533 (2009) (arXiv:0911.0931).

[4] G. Aad et al. (ATLAS Collab.), Phys. Rev. Lett. 105, 161801 (2010); ATLAS Collab., CONF-2010-093.

[5] CMS Collab., arXiv:1010.0203.

[6] S. Nussinov and R. Shrock, Phys. Rev. D 82, 034031 (2010).

[7] Indeed, if one were to envision fixing $\lambda$ at an arbitrarily high scale $\mu$, then the value of $\lambda$ at a low scale would approach zero, the property known as triviality.

[8] T. Appelquist, D. Karabali, and L. C. R. Wijewardhana, Phys. Rev. Lett. 57, 957 (1986); T. Appelquist, J. Terning, and L. C. R. Wijewardhana, Phys. Rev. Lett. 77, 1214 (1996); T. Appelquist, A. Ratnaweera, J. Terning, and L. C. R. Wijewardhana, Phys. Rev. D 58, 105017 (1998); T. Appelquist, K. Lane, and U. Mahanta, Phys. Rev. Lett. 61, 1553 (1988).

[9] R. Dashen, Phys. Rev. D 3, 1879 (1971); S. Weinberg, Phys. Rev. D 13, 974 (1976); M. Peskin, Nucl. Phys. B175, 197 (1980); J. Preskill, Nucl. Phys. 177, 21 (1981).

[10] In general, if one has fermions transforming according to the representations $R_{1}$ and $R_{2}$ of a gauge group, then a measure of the attractiveness of the condensation channel $R_{1} \times R_{2} \rightarrow R_{\text {cond }}$ is $\Delta C_{2}=C_{2}\left(R_{1}\right)+C_{2}\left(R_{2}\right)-C_{2}\left(R_{\text {cond }}\right)$, where $C_{2}(R)$ is the quadratic Casimir invariant for the representation $R$.

[11] This is to be contrasted with the situation in a strongly coupled chiral gauge theory, where the associated gauge symmetry does self-break [12].

[12] S. Raby, S. Dimopoulos, and L. Susskind, Nucl. Phys. B 169, 373 (1980).

[13] This suppression of low-energy amplitudes involving (P)NGB's is demonstrated experimentally via the success of low-energy pion theorems, as discussed, e.g., in S.
L. Adler and R. F. Dashen, Current Algebra (Benjamin, New York, 1968).

[14] L.-F. Li, Phys. Rev. D 9, 1723 (1974); V. Elias, S. Eliezer, and A. R. Swift, Phys. Rev. D 12, 3356 (1975).

[15] Although some of our discussion in this section is review, the bounded range for negative $\lambda_{2}$ in Eq. (5.26) and its consequences do not seem to have been noted before in the literature.

[16] S. J. Brodsky and R. Shrock, Phys. Lett. B 666, 95 (2008).

[17] Recent lattice simulations with fermions in the fundamental representation include T. Appelquist, G. Fleming, and E. Neil, Phys. Rev. Lett. 100, 171607 (2008); Phys. Rev. D 79, 076010 (2009); T. Appelquist et al., Phys. Rev. Lett. 104, 071601 (2010); arXiv:1009.5967; A. Deuzeman, M. P. Lombardo, and E. Pallante, Phys. Lett. B 670, 41 (2008); arXiv:0810.1719, arXiv:0904.4662; X.Y. Jin and R. Mawhinney, PoS Lattice-2008:059 (2008), arXiv:0812.0413 Z. Fodor, K. Holland, J. Kuti, D. Nogradi, and C. Schroeder, Phys. Lett. B 681, 353 (2009); arXiv:0911.2934, arXiv:0911.2463 A. Hasenfratz, arXiv:1004.1004 Recent reviews include T. Appelquist, Prog. Theor. Phys. 180, 72 (2009) and G. Fleming, talk at the Int. Conf. on High Energy Physics ICHEP 2010, Paris, http://www.ichep2010.fr

[18] J. M. Cornwall, R. Jackiw, and E. Tomboulis, Phys. Rev. D 10, 2428 (1974).

[19] T. Appelquist and R. Shrock, Phys. Rev. Lett. 90, 201801 (2003).

[20] T. Appelquist and J. Terning, Phys. Rev. D 50, 2116 (1994); T. Appelquist and R. Shrock, Phys. Lett. B 548, 204 (2002); T. Appelquist, M. Piai, and R. Shrock, Phys. Rev. D 69015002 (2004).

[21] T. A. Ryttov and R. Shrock, Phys. Rev. D 82, 055012 (2010).

[22] H. Georgi and S. Glashow, Phys. Rev. Lett. 32, 438 (1974).

[23] H. Fritzsch and P. Minkowski, Ann. Phys. 93, 193 (1975).

[24] S. Raby, in Particle Data Group, J. Phys. G 37, 193 (2010).

[25] E. Farhi and L. Susskind, Phys. Rev. D 20, 3404 (1979); N. D. Christensen and R. Shrock, Phys. Rev. D 72, 
035013 (2005); S. B. Gudnason, T. A. Ryttov, and F. Sannino, Phys. Rev. D 76, 015005 (2007); N. Chen and R. Shrock, Phys. Rev. D 78035002 (2008).
[26] T. A. Ryttov and R. Shrock, to appear. 